MS20-05

\section{Total Scattering at the X04SA beamline of SLS}

Antonio cervellino $^{1}$, Ruggero Frison ${ }^{2}$

1. Photon Science Division, Villigen, Switzerland

2. Department of Chemistry, University of Zurich, Switzerland \&

Excelsus Structural Solutions (Swiss AG), PARK innovAARE,

Switzerland, Zurich, Switzerland

\section{email: antonio.cervellino@psi.ch}

The X04SA Powder Diffraction beamline [1] has been developed with Total Scattering (TS) experiments at the forefront. The requirements for TS are perfectly compatible with maintaining the best capabilities for classical synchrotron XRPD, as the main TS requirement is - top data quality. The energy flexibility of the beamline $(5-30 \mathrm{keV}$ with high photon flux and sufficient detection efficiency) and the excellent detectors installed (a 2D Pilatus 6M and a large 1-D Mythen II) make up the rest, allowing for the required momentum transfer especially with the Mythen II [1]. In fact, collecting data to $140 \mathrm{deg}$ with the Mythen, the Qmax can reach $>28 \AA-1$, sufficient for most materials, while especial emphasis is given to reciprocal space TS (modelling the data directly from atomic coordinates via the Debye Scattering Equation, as per the DFA method [2]), where the absence of a direct Fourier transform of the data makes the need for high Qmax less stringent whilst the data quality must be even higher in all respects.

Increasing data quality is a process that involves the hardware - whereas all forms of parasitic scattering and all instrumental and intrinsic distortions (inelastic scattering, absorption,..) can be minimised and/or corrected whilst maximizing the sample scattering signal. The software plays also a critical role, inasmuch the information content of the data is fully preserved and the raw data are processed, corrected and subtracted [2] to yield precisely the desired part - the sample's elastic scattering pattern and its absolute scale sharpened counterpart $\mathrm{S}(\mathrm{Q})$ or the latter's direct space Fourier equivalent interatomic distance pair distribution function $\mathrm{G}(\mathrm{r})$.

The hardware (rotating capillary, liquid jet and suspended droplet) enhancements will be presented, together with the state-of-the-art, never before presented procedures and algorithms for calibrating the detector (pixel-wise positional and efficiency corrections [2]) together with the most effective and still partly unpublished algorithms and methods for data processing, merging, subtraction and correction - including a novel lossless smoothing-denoising [2] that permits to acquire high-quality low-noise background patterns in a fraction of the time previously required. Furthermore, subsequent processing [2] to obtain the absolute scale sharpened $\mathrm{S}(\mathrm{Q})$ and the $\mathrm{G}(\mathrm{r})$ functions in a reliable and automated way are presented and compared with existing dedicated softwares like (GudrunX [3], PdfGetX3 [3].

As an outlook, simultaneous Quantitative Phase Analysis in $\mathrm{Q}$ and $\mathrm{r}$ spaces - thus made independent from the crystallinity of components - is envisaged.
References:

[1] Willmott, P.R. et al., (2013) J. Synchrotron Rad., 20, 667-682; Bergamaschi A. et al., (2010), J. Synchrotron Rad., 17, 653-668.

[2] Cervellino A. et al., J. Appl. Cryst. 2015, 48, 2026-2032; J.

Comput. Chem. 2006, 27, 995-1008; J. Appl. Cryst. 2014, 47, 1755 -

1761; Cervellino A., Frison R. (2018), unpublished

[3] Soper Alan K. and Barney Emma R., (2011 ). J. Appl. Cryst., 44, 714-726; Juhàs, P. and Davis, T. and Farrow, C. L. and Billinge, S.J.L., (2013). J. Appl. Cryst. 46, 560-556

Keywords: Synchrotron XRPD, Total Scattering, Data processing 